\title{
Diseño de sistemas productivos y mejora de su eficiencia mediante técnicas de realidad virtual
}

\author{
Design of productive systems and improves of their efficiency with \\ techniques of virtual reality \\ José Manuel Arenas Reina', Manuel García García', Miguel Ángel Sebastián Pérez² y Alberto \\ Sánchez Lite ${ }^{3}$
}

I E.U.I.T Industrial de la Universidad Politécnica de Madrid.

2 E.T.S. Ingenieros Industriales de la Universidad de Valladolid.

${ }^{3}$ E.T.S. Ingenieros Industriales de la UNED, Madrid.

josem1960@wanadoo.es

Fecha de recepción: 09-11-09

Fecha de aceptación: 03-03-10

Resumen: El presente trabajo expone una nueva metodología para una adecuada utilización de herramientas virtuales $3 \mathrm{D}$ en procesos de fabricación y montaje. El sistema toma como referencia el modelo 3D del producto para generar el correspondiente entorno virtual 3D donde se diseña y valida el proceso de manera interactiva. Este sistema es capaz de generar todos los recursos necesarios para la realización del proceso de montaje, desde el diseño de la estación de trabajo hasta la incorporación del factor humano pasando por los factores productivos y ergonómicos. La aplicación práctica de esta metodología facilita una rápida adaptación a los posibles cambios del producto y favorece la mejora continua del proceso.

Palabras clave: Realidad virtual, diseño del proceso, mejora de métodos, ergonomía.

Abstract:The present work exposes a new methodology for an appropriate use of virtual tools 3D in manufacturing and assembly processes. The system uses the product design in 3D to generate the 3D virtual environment. In this environment, the process is designed in an interactive way. This system is able to generate all the necessary resources for the realization of the assembly process, from the design of the work station until the incorporation of the human factor going by the productive and ergonomic factors. The practical application of this methodology allows a quick adaptation to the possible changes of the product and facilitates the continuous improvement of the process.

Key Words: Virtual reality, process design, process improvement, ergonomics.

\section{Introducción}

Tradicionalmente se han considerado dos tendencias relevantes y hasta cierto punto contrapuestas en el ámbito de la metodología de diseño de productos (Ferrer, l., 2003). Por un lado, se proponen modelos descriptivos que no plantean procedimientos sistemáticos (Cross, N., 1994) y, por otra parte, se consideran modelos prescriptivos que, teniendo en cuenta una clara identificación del objetivo, generan un procedimiento sistemático para cumplir las especificaciones fijadas (Das, B. y Sengupta, A. 1996). En ambas metodologías de diseño la implementación del producto es una etapa clave que requiere un uso adecuado de la tecnología y los procesos de fabricación que permita lograr las especificaciones técnicas y económicas previstas. Para ello, es necesario un enfoque integrador que incorpore los condicionantes del proceso de fabricación en las fases iniciales del proceso de diseño del producto. Aunque los primeros trabajos en esta línea datan de la Segunda Guerra Mundial, los resultados más eficientes se obtienen en la década de los 80 con la aparición de una incipiente ingeniería simultánea junto a las herramientas de diseño para el montaje. Este enfoque se completa posteriormente con la ingeniería concurrente (que tiene en cuenta todas las etapas del ciclo de vida) y la ingeniería simultánea y colaborativa (que incorpora los condicionantes y relaciones del resto de los departamentos de la empresa).

La ergonomía ha sido utilizada tradicionalmente para disminuir el número de lesiones ocupacionales tratando de descubrir las posturas y tareas que crean tensiones músculo - esqueléticas significativas. Sin embargo, los principios que son la base de la ergonomía se pueden utilizar también para mejorar potencialmente la productividad. Las guías ergonómi- 
cas pueden permitir la predicción de esas posturas y ayudar al diseño del lugar de trabajo de forma que se maximice el rendimiento de los trabajadores (Resnick, M.L. y Zanotti, A., 1997).

La ingeniería ergonómica y de factores humanos aplica la información sobre el comportamiento, las capacidades y las limitaciones del ser humano en el diseño del puesto (máquinas, útiles, herramientas, etc.) y proceso de trabajo (actividades, ambiente de trabajo, etc.) para conseguir que el operario realice su labor de manera segura, productiva y eficiente (Chapanis, A., 1985). La fabricación industrial es un sistema complejo donde intervienen los factores humanos, las máquinas, el ambiente de trabajo y el modelo organizativo concreto. Para que este sistema complejo funcione eficazmente, la dirección debe asegurar el funcionamiento óptimo de cada uno de los componentes del mismo. Desde el punto de vista ergonómico, el objetivo es ajustar la tarea al ser humano y no el ser humano a la tarea (Grandjean, E., 1982).

Muchos estudios han mostrado los efectos positivos de aplicar los principios de la ergonomía en los puestos de trabajo, medicina, seguridad y salud ocupacional, diseño de máquinas, sistemas de trabajo, ambiente de trabajo e instalaciones (Grandjean, E., 1982; Konz, S., I 983; Sanders, M.S. and McCormick, E.J., I 992; Shikdar, A. and Das, B., 1995; Das, B. and Sengupta, A., 1996; Das, B. and Shikdar, A., 1999). El uso eficaz de la ergonomía en el diseño del sistema del trabajo puede lograr un equilibrio entre las características del trabajador y las demandas de la tarea y, de este modo, se puede incrementar la productividad del trabajador mejorando su seguridad, bienestar físico y mental así como su satisfacción profesional.

Los estudios ergonómicos han dado como resultado bases de datos y guías para aplicaciones industriales. Sin embargo, su aplicación en fabricación industrial es todavía limitado y tiene un bajo nivel de aceptación. Actualmente y en la mayoría de los sectores industriales, el diseño del sistema de trabajo no responde a un planteamiento general sino que el análisis suele centrarse en mejoras puntuales relativas a máquinas, útiles y herramientas que mejoren la productividad (Das, B., 1987; Sanders, M.S. and McCormick, E.J., I992; Shikdar, A. and Das, B., 1995). Sin embargo, en el ámbito del montaje manual se han desarrollado una gran cantidad de procedimientos para el diseño y la planificación del puesto (Bullinger, H. J., 1986; Braun, W.J. et al., 1996). Un aspecto básico en la planificación de estos procedimientos es la selección de la herramienta y su ajuste en el lugar de trabajo con consideraciones económicas y ergonómicas. Estos proce- dimientos son semejantes y plantean orientaciones generales pero no incluyen normas o información detallada que permita implementar las diferentes fases del diseño de puesto (Braun, W.J. et al., 1996).

El diseño de puestos de trabajo industriales se considera un proceso iterativo y altamente interactivo. El diseñador tiene que considerar numerosas restricciones y diversas soluciones para objetivos contradictorios (Evans, S.M. and Chaffin, D.B., 1986). Empresas de diferentes sectores utilizan herramientas de modelado del ser humano para analizar y visualizar sus productos. Los departamentos implicados en el proceso de diseño del producto utilizan diferentes herramientas para representar a usuarios directos, usuarios secundarios, operarios de la planta, etc. Estas herramientas permiten incorporar al diseño los diversos aspectos de la interacción producto - ser humano con enfoques relativos a condiciones ambientales y físicas, tales como la geometría y las fuerzas. Así, por ejemplo, en el sector del automóvil se utilizan herramientas de modelado del ser humano para evaluar en la etapa de diseño la idoneidad del interior del vehículo a las características humanas.

Tanto los responsables de desarrollo y revisión (Porter, J.M. et al., 1993; Porter, M., 1998; Chaffin, D., 200I) como los usuarios (Bowman, D., 200I) de dichas herramientas de simulación aseguran que su empleo reduce tiempo y costes de desarrollo de los productos ya que, por una parte, las herramientas de modelado posibilitan la realización de pruebas rápidas (al comprobar más rápido los diseños preliminares o más bastos se acelera fase inicial del diseño) $y$, por otro lado, estas herramientas proporcionan medios para desarrollar una metodología estandardizada de evaluación (Bowman, D., 200I) con potencial para convertirse en un medio de validación estándar y método de referencia (Sundin, A., 200I). Por todo ello, diseñadores e ingenieros de producto y proceso consideran que estas herramientas son de gran utilidad para realizar su labor profesional (Haslegrave, C.M. and Holmes, K., 1994). Así, la industria automovilística, siempre en la vanguardia tecnológica, emplea dichas herramientas de simulación humana aunque con un enfoque parcial (Blomé, M. et al., 2003) y un limitado soporte documental (Hanson, L. et al., 2006).

Tradicionalmente, las interacciones «producto-ser humano» han sido evaluadas en etapas del proceso relativamente tardías mediante el empleo de maquetas físicas (Porter, J.M. et al., 1993). Cuando la integración de los factores humanos se realiza en experimentos del laboratorio y/o de campo suponen 
un elevado coste y mucho tiempo de dedicación (Helander, M.G., 1999). Para evitar estos inconvenientes y durante los últimos 20 años, se ha incrementado la utilización de herramientas de modelado del ser humano que facilitan las evaluaciones ergonómicas en ambientes virtuales reduciendo, con ello, el número de comprobaciones físicas.

Existen dos vías para la construcción de modelos simulados. Por un lado es posible la programación directa, utilizando un lenguaje de programación y, por otro, existe la posibilidad de utilizar un paquete de simulación específico. Junto a estas dos alternativas han surgidos sistemas híbridos. Estos sistemas, utilizando entidades y conexiones ya creadas, permiten elaborar modelos de forma rápida, que junto con la posibilidad de la programación informática adicional, hacen posible la reproducción de modelos más complicados.

El presente trabajo analiza y muestra el método para una adecuada utilización de estas herramientas en un proceso de montaje típico del sector del automóvil. El método desarrollado toma como referencia el modelo 3D del producto para generar el correspondiente entorno virtual 3D donde se diseña y comprueba el proceso de manera interactiva. Los resultados obtenidos han permitido generar un entorno virtual 3D del montaje en el que producto y proceso interactúan facilitando una rápida adaptación a los posibles cambios y favoreciendo la mejora continua del proceso. La aplicación de esta metodología en una empresa del sector del automóvil ha permitido reducir un 15\% el tiempo de montaje así como disminuir un $90 \%$ las lesiones músculo-esqueléticas derivadas de posturas inadecuadas en el desarrollo del trabajo.

\section{Descripción de la metodología}

La metodología que se desarrolla en este trabajo se basa en la generación de un entorno virtual 3D que tiene como referencia el modelo 3D del producto e incluye y valida el proceso de montaje de forma virtual. Asimismo genera todos los recursos necesarios para la realización del proceso de fabricación, desde el diseño de la estación de trabajo hasta la incorporación del factor humano pasando por los factores productivos y ergonómicos.

Con la aplicación de esta metodología se pretende incorporar los factores humanos en las primeras etapas de diseño y crear una herramienta que pueda ser utilizada en las fases de iniciales de creación de un producto para poder evaluar, de una forma detallada, las ventajas e inconvenientes del nuevo producto de manera general tanto en el montaje como en la fabricación. Esta metodología puede ser realimentada en las distintas fases de vida del producto, desde el diseño y desarrollo hasta la fabricación.

Se trata de una aproximación sistemática al desarrollo y diseño de procesos, que ha dado como fruto un modelo conceptual que se ha aplicado en una situación real de una multinacional fabricante de piezas para la automoción. Este planteamiento permite mostrar las modificaciones que una línea de producción sufre debidas a la incorporación de un producto completamente distinto al que actualmente se realiza. Posee como principal ventaja la modificación de los procesos de montaje para simplificar y reducir operaciones y adaptar el montaje de todos los elementos al factor humano.

Como ya se ha indicado, partiendo de la geometría CAD 3D se estudia el montaje, los factores humanos, los tiempos de montaje, la distribución de puestos, creación de puestos de montaje, diseño de líneas en 3D, etc. De este modo se desarrolla una metodología en las que se implementan las limitaciones teóricas de la interrelación hombre - sistema, demostrando la validez del modelo planteado, teniendo en cuenta los factores personales respecto a las capacidades físicas: factores físicos (respecto al manejo de cargas y realización de actividades repetitivas), factores ambientales, diseño de recursos y medios de montaje, diseño del puesto de trabajo y disposición de recursos, seguridad, creación de procedimientos y guías de entrenamiento para los operarios. La aplicación de herramientas de simulación muestra la validez de la metodología sistemática para incorporar los factores humanos en el diseño virtual de una línea de fabricación, visualiza la factibilidad del montaje del nuevo producto y permite la reducción de tiempos y costes de diseño del producto y proceso.

\section{Aplicación práctica en un taller de fabricación y montaje}

El uso de entornos virtuales en el montaje manual de componentes convierte a estas herramientas en una verdadera ingeniería simultánea donde las variables inherentes al factor humano y la productividad interactúan.

La aplicación que se presenta analiza el montaje de los componentes de un salpicadero de vehículo. Este conjunto incorpora una gran variedad de piezas 
que deben ser montados antes de introducir el salpicadero en el interior del vehículo.

La aplicación de la metodología descrita en el apartado anterior se ilustra en la Figura I. Según los datos de partida y las primeras concepciones de producto, junto con los requerimientos del cliente y las experiencias de otros proyectos se define una célula de montaje prototipo en un entorno de trabajo virtual 3D, donde se identifica el producto y los elementos necesarios para el realizar el ensamble y todas y cada una de las operaciones que constituyen el montaje final.

La Figura I representa la metodología seguida desde las primeras etapas, pasando por la generación de un puesto de trabajo virtual 3D en el que se realizan todas las operaciones de montaje en una sola célula, para pasar a una etapa en la que teniendo en cuenta las restricciones de productividad exigidas se desarrolla una línea de montaje virtual 3D con la distribución de todos las operaciones de montaje en los distintos puesto que aseguran el cumplimiento del tiempo de ciclo necesario para alcanzar la producción marcada. En este punto es posible generar una serie de ensayos que permitan observar el comportamiento de la línea ante situaciones como errores, paradas, averías y aumentos de producción. Toda la información obtenida en estas fases de simulación virtual 3D (información que en cada una de las fases de vida del proyecto se ha realimentado con las modificaciones de producto necesarias para el cumplimiento funcional y el cumplimiento productivo) potencia la entrada en el pre-diseño mecánico de la línea con el lanzamiento de los Cuadernos de Especificaciones para la creación o compra de la línea final. Teniendo en cuenta estos resultados, el marco para una ingeniería colaborativa resulta mucho más sencillo con la ayuda de este tipo de herramientas.

La aplicación que se presenta en este trabajo se centra en el diseño de las estaciones de trabajo y tiene en cuenta los factores productivos y el factor humano. Este último mediante un enfoque ergonómico basado en un estudio postural de todas las operaciones de montaje de los componentes que integran el salpicadero del vehículo.

El estudio postural se ha realizado mediante la herramienta SAFEWORK integrada en el entorno de Catia V5. Para la valoración ergonómica se utilizaron métodos indirectos integrados en las herramientas de simulación RULA y NIOSH LE. Dicho estudio postural, planteado para el diseño de la línea de montaje, consta de dos etapas y se resume en la Figura 2.

Se determinaron las alturas máximas y mínimas de la zona de trabajo mediante el estudio postural y de cargas con los percentiles $95 \%$ hombre y $5 \%$ mujer. Se realizaron estudios de altimetrías para asegurar posturas y movimientos que debe realizar el opera-

Figura I

Metodología de generación para un entorno virtual de montaje 3D

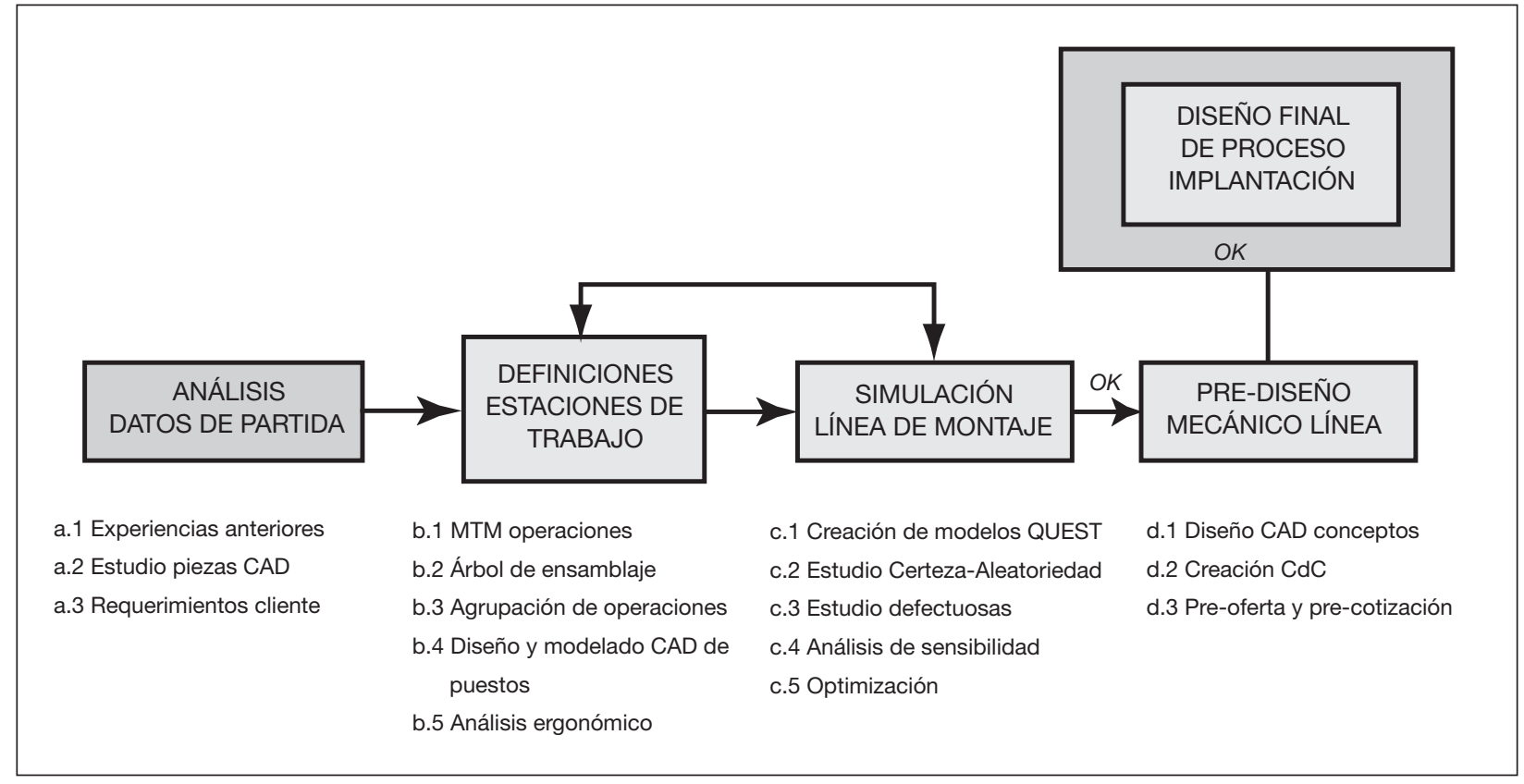


Figura 2.

Metodología utilizada para el diseño ergonómico de pospuesto de montaje.

\begin{tabular}{|c|c|}
\hline - Geometría CAD 3D Producto \\
- Proceso de Montaje
\end{tabular}

rio para realizar el montaje con un nivel aceptable desde el punto de vista ergonómico. Asimismo se diseñó un utillaje que permitiera girar la estructura base para el montaje los componentes, de tal forma que los ángulos de inclinación del componente principal montado en el utillaje facilitaran un montaje adecuado desde el punto de vista ergonómico. Algunos de los resultados se reflejan en la Figuras 3 y 4.

El desarrollo de este estudio permitió tener en cuenta el factor humano en las primeras etapas de diseño, generando modificaciones de producto que permitieron tener en cuenta los factores de productividad y la seguridad en el desarrollo de la ingeniería de fabricación.

\section{Resultados y conclusiones}

El presente trabajo analiza y muestra un método para una adecuada utilización de herramientas virtuales $3 \mathrm{D}$ en un proceso de montaje típico del sector del automóvil. El sistema diseñado integra el proceso productivo en la concepción del producto generando la adecuada realimentación. De este modo se aborda la generación de un nuevo producto mediante una metodología de diseño producto - proceso que engloba todas las etapas del ciclo de vida del mismo, que permite una rápida adecuación a las necesidades del cliente y a la situación del mercado en el marco de la competencia industrial actual.

Figura 3.

Análisis Ergonómico de la céulal de trabajo elemento 7 percentil Hombre 95\%

\begin{tabular}{|c|c|c|c|}
\hline \multirow{9}{*}{ 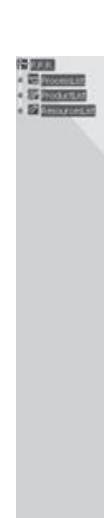 } & \multicolumn{3}{|c|}{ Rutranayssis } \\
\hline & $\begin{array}{l}\text { Side: } \otimes \text { Left } \\
\text { Parameters }\end{array}$ & & \\
\hline & $\begin{array}{l}\text { Posture } \\
\text { S Static Intermitent }\end{array} \mathrm{O}_{\text {Repested }}$ & & $\begin{array}{l}+ \text { Iupper Amm: } \\
+ \text { Frearmm }\end{array}$ \\
\hline & 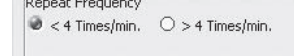 & & $\begin{array}{l}+1 \text { Whist: } \\
\pm \text { Wrist Twist: }\end{array}$ \\
\hline & 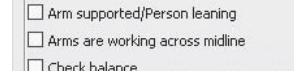 & & $\begin{array}{ll}\text { Muscle: } & 0 \\
\text { Farceload: } & 0\end{array}$ \\
\hline & Lood: : مokg & & $\begin{array}{l}\text { IN Neck: } \\
+ \text { Iruph: }\end{array}$ \\
\hline & $\begin{array}{l}\text { Score } \\
\text { Final Score: } 1 \\
1\end{array}$ & $<<$ less & $\begin{array}{ll}\text { Leg: } & 1 \\
\text { Posture B: } & 1=\end{array}$ \\
\hline & & & Neck, Trunk and Leg: 1 = \\
\hline & - & & 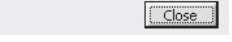 \\
\hline
\end{tabular}


Figura 4

Detalle de la célula de trabajo

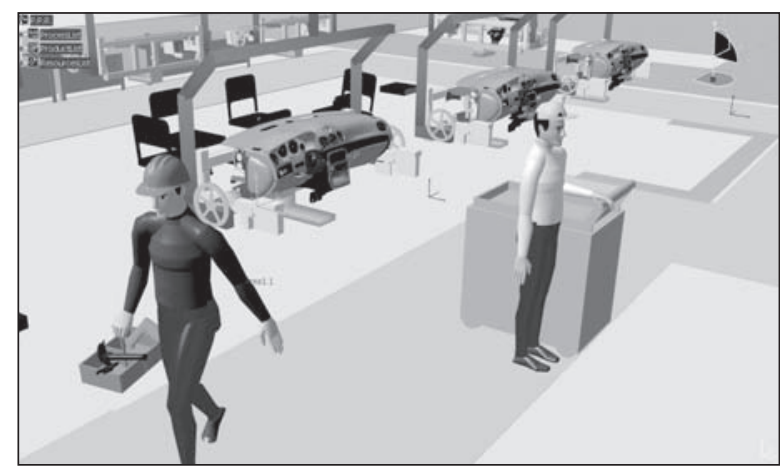

El método desarrollado toma como referencia el modelo 3D del producto para generar el correspondiente entorno virtual 3D donde se diseña y valida el proceso de manera interactiva. Este sistema es capaz de generar todos los recursos necesarios para la realización del proceso de montaje, desde el diseño de la estación de trabajo hasta la incorporación del factor humano pasando por los factores productivos y ergonómicos. Los resultados obtenidos han permitido generar un entorno virtual 3D del montaje en el que producto y proceso interactúan facilitando una rápida adaptación a los posibles cambios y favoreciendo la mejora continua del proceso. La aplicación de esta metodología en una empresa del sector del automóvil ha permitido reducir un 15\% el tiempo de montaje así como disminuir un $90 \%$ las lesiones músculo-esqueléticas derivadas de posturas inadecuadas en el desarrollo del trabajo. Asimismo se disminuyen los costes resultados del análisis de las diferentes alternativas de diseño y fabricación.

Así pues, el método desarrollado optimiza el diseño del proceso mediante el uso de herramientas virtuales que permiten incorporar de manera interactiva todos los factores relevantes del ciclo de vida del producto, mejorando la ingeniería de producto y del proceso y facilitando la introducción de cambios y mejoras.

\section{Referencias}

BLOME, M.; DUKIC, T.; HANSON, L.; HOGBERG, D (2003). «Computer-based protocol for human simulation report», In: Proceedings of IEA, Seoul, South Korea, Vol. 3, p. 30-33.

BOWMAN, D. (200I). «Using digital human modeling in a virtual heavy vehicle development environment», In: Chaffin, D. (Ed.), Digital Human Modeling for Vehicle and
Workplace Design. Society of Automotive Engineers, Inc., Warrendale, USA.

BRAUN,W.J;; REBOLLER, R.; SCHILLER, E.F. ( 1996). «Computer aided planning and design of manual assembly systems», International Journal of Production Research, Vol. 34(8), p. 2317-2333.

BULLINGER, H. J. (Ed.) (1986). «Systemtische montageplanung», Munich: Hanser.

CHAFFIN, D. (200I). «Digital Human Modeling for Vehicle and Workplace Design. Introduction», In: Chaffin, D. (Ed.), Society of Automotive Engineers, Inc., Warrendale, USA.

CHAPANIS, A. (1985). «Some reflections on progress», Proceedings of the Human Factors 29th Annual Meeting, Santa Monica, CA, USA, p. I-8.

CROSS, N. (1994). «Engineering design methods: strategies for production design», Chichester, John Willey \& Son, New York.

DAS, B. (1987). «An ergonomic approach to designing a manufacturing work system», International Journal of Industrial Ergonomics, Vol. I (3), p. 23 I-240.

DAS, B. SENGUPTA, A. (1996). «Industrial workstation design: a systematic ergonomic approach» Applied Ergonomics, Vol. 27(3), p. I57- 163.

DAS, B. SHIKDAR, A. ( 1999). «Participative versus assigned production standard setting in a repetitive industrial task: a strategy for improving worker productivity. International Journal of Occupational Safety and Ergonomics, Vol. 5(3), p. 4l 7-430.

EVANS, S.M.; CHAFFIN, D.B. (1986). «Using interactive visual displays to present ergonomic information in workplace design», In W. Karwowski, A. M. Genaidy, \& S. S. Asfourm (Eds.), Trends in ergonomics/human factors III, Amsterdam: Elsevier, p. 17-24.

FERRER REAL, I. (2003). «Contribuciones metodológicas para fabrica», Tesis Doctoral. ISBN 978-84-69077। 5-3. Gl7I5-3.

GRANDJEAN, E. (1982). «Fitting the task to the man: an ergonomic approach», Taylor \& Francis, London.

HANSON, L.; BLOME, M.; DUKICC, T.; HOGBERGE, D. (2006). «Guide and documentation system to support digital human modeling applications», International Journal of Industrial Ergonomics, Vol. 36, p. 17-24

HASLEGRAVE, C.M.; HOLMES, K. ( 1994). «Integrating ergonomics and engineering in the technical design process», Applied Ergonomics, Vol. 25 (4), p. 21 I-220

HELANDER, M.G. ( 1999). «Seven common reasons to not implement ergonomics», Inter. J. Ind. Ergon. Vol. 25(I), p. 97-101

KONZ, S. ( 1983). «Work design: industrial ergonomics $\left(2^{\text {nd }}\right.$ ed. $) »$, Grid Columbus, Ohio. 
OOTERMAN, B. J. (200 I). «lmproving product development projects by matching product architectures and organization», Tesis Doctoral, University of Gröningen, The Netherlands.

PORTER, J.M.; CASE, K.; FREER, M. T.; BONNEY, M.C. (1993). «Computer-aided ergonomics design of automobiles», In: Peacock, B.,Karwowski,W. (Eds.), Automotive Ergonomics, Taylor \& Francis, London.

PORTER, M. (1998). «Justifying the incorporation of ergonomics into organisational strategy-beyond single issue solving», In: Kumar, S. (Ed.), Advances in Occupational Ergonomics and Safety, IOS Press, Amsterdam,Vol. 2, p. $119-122$.
RESNICK, M.L.; ZANOTTI, A. (1997). «Using ergonomics to target productivity improvements», Computers Industrial. Engineering, Vol. 33 ( I-2), p. I85-188.

SANDERS, M.S.; MCCORMICK, E.J. (1992). «Human factors in engineering and design (7th ed.)», McGraw-Hill, New York.

SHIKDAR, A.; DAS, B. (1995). «A field study of worker productivity improvements», Applied Ergonomics,Vol. 26(I), p. $21-27$.

SUNDIN, A. (200I). «Participatory ergonomics in product development and workplace design», Doctoral Thesis, Chalmers University of Technology, Gothenburg, Sweden. 